\title{
Recent Development of SANS BATAN Spectrometer (SMARTer) in Serpong for Simultaneous and Automatic Measurements
}

\author{
E.G.R. Putra , Bharoto, N. Suparno, Sairun and E. Santoso \\ Center for Technology of Nuclear Industry Materials, National Nuclear Energy Agency, \\ Puspiptek Area, Serpong, Tangerang 15314, Indonesia
}

\section{ARTICLE INFO}

Article history:

Received 08 November 2013

Received in revised form 23 December 2013

Accepted 27 December 2013

Keywords:

SANS spectrometer

Programmable motor controller

Data acquisition

in situ experiment

\begin{abstract}
A B S T R A C T
The 36 meter SANS BATAN spectrometer (SMARTer) in Serpong Indonesia has been developed for simultaneous and automatic measurements. The existing motor controller of ISA (Industry Standard Architecture) interface was replaced by the programmable motor controller of PCI (Peripheral Component Interconnect) interface, since it has speed and interrupt-sharing advantages, to drive all stepper motors for collimator, pinhole, and detector movements of the spectrometer. The recent development makes all motors moved and controlled simultaneously in setting up the instrument configuration before performing the experiment. Along with that improvement, the data acquisition software has been also developed to drive the beam stopper movement in two directions as well as to read the absolute collimator and detector positions, to acquire neutron counts on the monitor and the main detector, and also to control automatically up to 12 sample positions of the sample changer. The collected neutron counts is displayed in real time on the main monitor window, and the counts is saved in a special format for further data reduction and analysis. The developed data acquisition software has been implemented and performed for experiment that use preset time or preset count mode, and the automatic sample changer
\end{abstract}

(C) 2013 Atom Indonesia. All rights reserved

\section{INTRODUCTION}

A small angle neutron scattering (SANS) is very suitable method for investigating the static and dynamic-structures of wide range materials such as alloys, ceramics, polymers, colloids, liquid crystals as well as biological materials in the range scale of 1 - 100 nanometer. Therefore, it is understandable that many SANS spectrometers are currently in operation, designed or constructed at reactor and spallation facilities or have just been upgraded [1-3].

The 36 meter SANS spectrometer (SMARTer) in Serpong, Indonesia which is one of the largest SANS spectrometers nowadays in the Asia-Oceania region was constructed in the neutronguide hall (NGH) of the $30 \mathrm{MW} \mathrm{G}$. A. Siwabessy reactor in 1992 [4]. Figure 1 shows the overview of SMARTer. This spectrometer consists of a mechanical velocity selector, an $18 \mathrm{~m}$-long collimator tube, a sample table and an $18 \mathrm{~m}$-long flight tube. The collimator system comprises of four sections of movable guide-tubes and one section of fixed collimator (non-reflecting tube or boron-

${ }^{*}$ Corresponding author.

E-mail address: giri@batan.go.id rubber tube), and pinholes. Meanwhile, the flight tube accommodates a $128 \times 128$ channel of two-dimensional ${ }^{3} \mathrm{He}$ position sensitive detector (2D-PSD). The detector can be moved continuously from $1.3 \mathrm{~m}$ to $18 \mathrm{~m}$ from the sample position and can also be shifted in the lateral direction for $0.1 \mathrm{~m}$ to increase the momentum transfer, $Q$-range. Variations of collimator length, pinhole and sampleto-detector distance (SDD) setup are fully controlled by a dedicated computer.

Due to the original main computer IBM PS2/70 used for handling all the motor drivers and for acquiring the neutron counts from the main detector, 2D-PSD had broken down in 2003, an inhouse project was proposed to put the spectrometer back in operation and work functionally [5]. This project was designed to upgrade gradually the system, i.e. electronics, mechanics, computer software for main control and data acquisition system, including the data reduction and analyses. In the beginning, the ISA (Industry Standard Architecture) motor controller interface was employed in the main control system of the spectrometer to control all the motor drivers, while General Purpose Interface Bus (GPIB) interface 

(b) Pre-sample flight path Collimation length $1.5,4,8,13$, and 18 meter from sample position

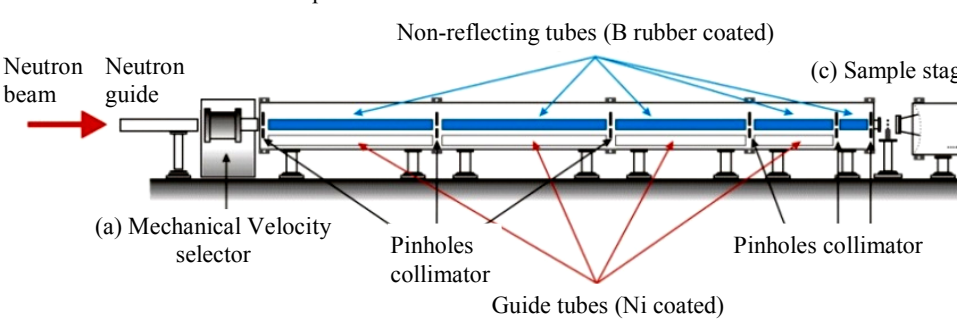

(d) Post-sample flight path detector position 1.3 to 18 meter continously from sample position

(g) Data acquisition system

Fig. 1. A Schematic drawing of the $36 \mathrm{~m}$ SANS BATAN spectrometer (SMARTer) in Serpong.

which employed in the data acquisition system was used to collect the neutron counts from the main 2-dimensional detector at a separated personal computer (PC). Those initial developments on the control and data acquisition systems have been working properly for performing the experiments [6,7]. Many experimental research works have been carried out using SMARTer and reported elsewhere [8-10].

Seeing as the number of local and regional users to utilize SMARTer had been increasing significantly in the last several years, then expanding the applicable condition and performance of spectrometer has to be taken. A major development has been completed by upgrading the main control system using PCI (Peripheral Component Interconnect) programmable motor controller for simultaneous setup configuration, employing automatic sample changer system for automatic sequence of measurements and improving the data acquisition system for handling all new available experiment modes. In this paper, we report the latest of development and the experimental results on the new implemented system.

\section{EXPERIMENTAL METHODS}

\section{Hardware and software development}

\section{Upgrading the main control system}

A motor controller software to control all motor drivers of the spectrometer operated via ISA interface was applied in 2005, Fig. 2a [6]. This control system handled 8 stepping motors of 4 collimator/neutron guide tubes, 6 stepping motors of six pinhole discs (each has 8 different configurations), 2 stepping motors of beam stop (XY movement directions) and 1 stepping motor of main detector for 1-direction movement. The feedback from encoder that providing status of all instrument devices is still read by a dedicated computer for device control system via the original RS232C interface connection through the CPU system in control panel. Meanwhile, another dedicated computer running on MS Windows ${ }^{\mathrm{TM}}$ has been also set for acquiring neutron counts from the 2-dimensional main detector via GPIB connection. However, the ISA interface card did not have a programmable IC (integrated circuit) which means that it sent pulses directly from the computer software to handle the motor driver and only worked properly when there is no any interruptions. Consequently, the control system could not handle all motor drivers through ISA interface and simultaneously read the device control status from encoders through the RS232C interface.

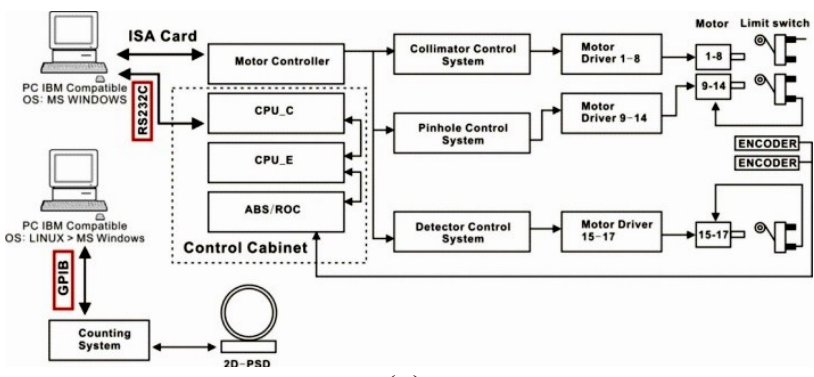

(a)

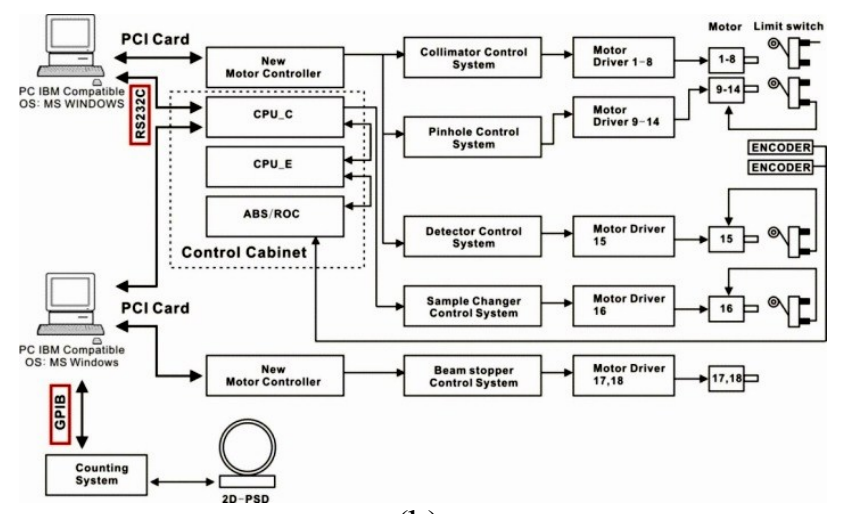

(b)

Fig. 2. The main control and data acquisition system applied in SMARTer : (a) 2005 - 2011 and (b) from 2011.

A new programmable motor controller (PMC) system through PCI interface card was developed and then implemented in 2011 to replace the previous system using ISA interface card, Fig. $2 b$. Each programmable motor controller card can 
handle up to 8 axes and worked independently, it means that 8 motors can be moved and controlled simultaneously. Two PCI interface cards were employed at the main control system computer to handle 15 motor drivers. This development has significantly reduced the consuming time for setting up the instrument configuration before running the experiment. The beam stopper control system was removed from the previous system and installed on the data acquisition computer using 4 axes PMC card in order to place the beam stopper in the direct beam while the pre-experiment is running simultaneously, Fig. 2b. The data acquisition computer also handles the sample changer driver system using a serial port (RS232C) and reads the equipment status from the encoders.

Due to the replacement of the motor controllers, the software for controlling all devices had also been developed. However, the command menu of the control system software had not been entirely modified [11], Fig. 3. The modification was only in the source code that makes all devices can be handled and controlled simultaneously, and the flowchart of the software of the main control system is shown in Fig. 4. In the first step, the new modified control software initializes the PMC card, reads the data $\log$ file which contains the information of the last setting configuration of all devices, and then resets the controller. In the previous software, it gave the user a preference for repositioning the setting configuration of the six pinholes and the beam stopper. This action embedded in the initial step for confirming those device positions since unexpected stop (emergency stop, power failure, etc.) might be occurred during the movement and caused some errors in the software. In spite of that, in the new modified software, this function has been deleted so the user can move the 6 pinhole discs to the reference position after the software is loaded. The next step is reading the pulses transferred directly from 13 encoders ( 8 in the four-sections of the collimator system, 1 in the main detector and 4 in the beam narrower systems). Those pulses converted into the position of the system are written to the PMC card and then displayed in the command menu of main window. A timer object has been used to read all the encoders and motor positions for every second that updates the status of all devices. Finally the software is ready to control each device by clicking the appropriate button, Fig. 3. According to the moving command, the software sends the moving function to PMC card, and makes a loop to check the device position whether it has reached the target position or has not. This loop will not make the software stall, since each device has its own timer which works independently. All of the moving results are saved in the file for the last status configuration of all devices, as well as in the usage data $\log$.
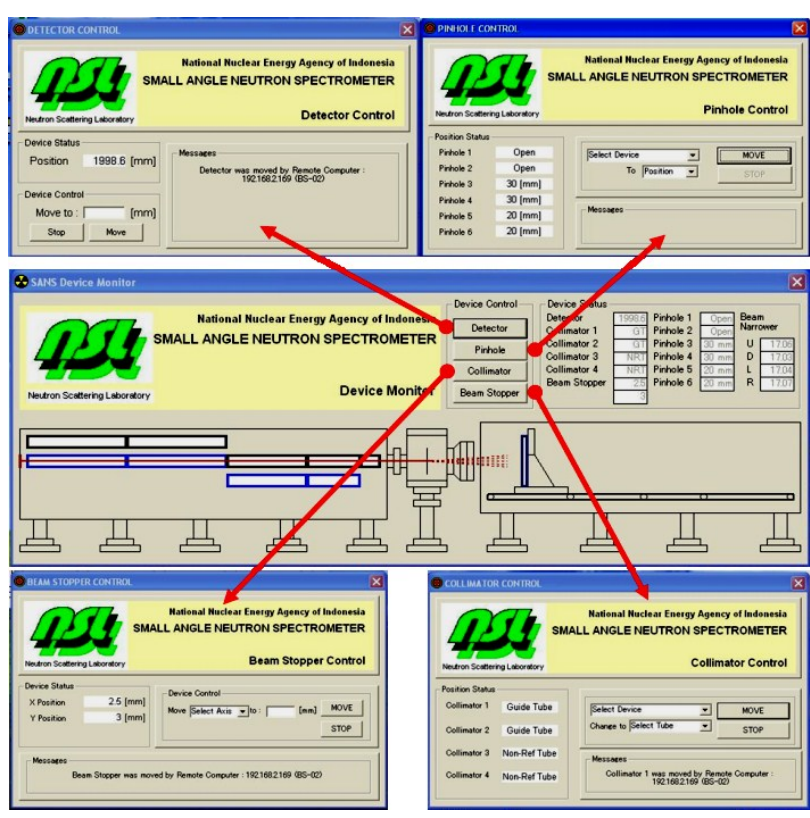

Fig. 3. The Graphical User Interface (GUI) in main monitor and control menu windows of the SMARTer at the main control system computer.

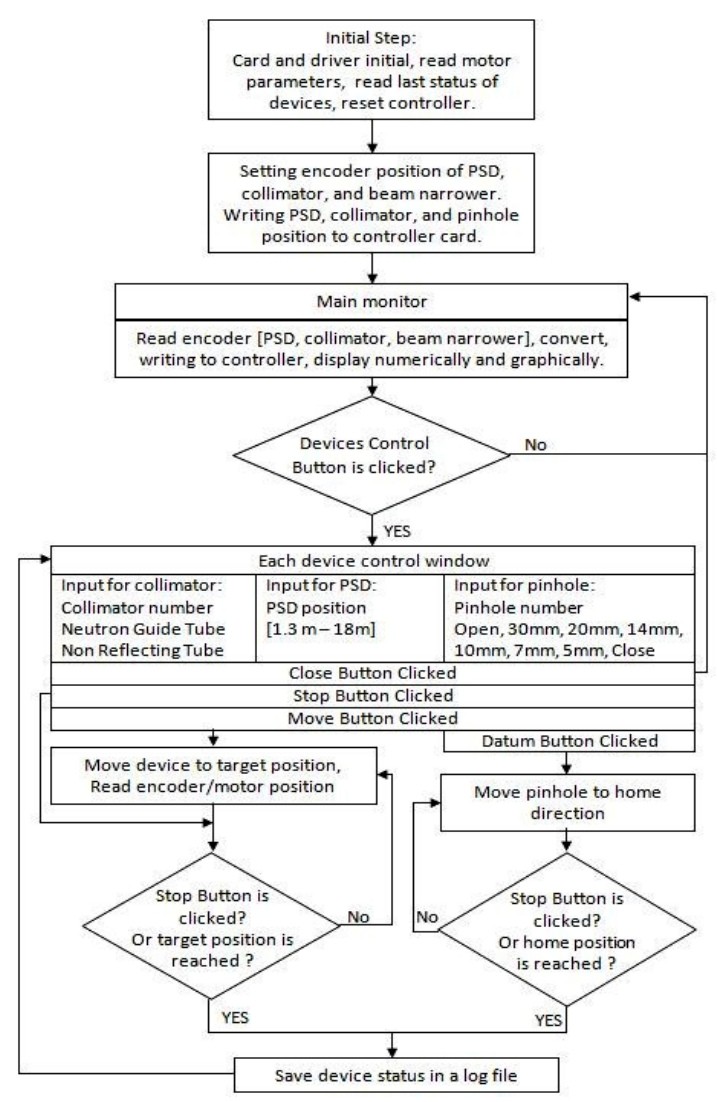

Fig. 4. Flowchart of the SMARTermain control system software implemented at the computer main. 


\section{Improving the data acquisition system}

Along with the development of the main control system, the new data acquisition software under MS Windows ${ }^{\mathrm{TM}}$ operating system is also developed to drive the beam stopper in two directions ( $\mathrm{x}$ and $\mathrm{y}$ ), to read the equipment status such as positions of the collimators, pinholes and the main detector, to acquire neutron counts on monitor detector and the main detector, and also to manage automatically up to 12 sample positions of the sample changer, Fig. 5. The new modified data acquisition software starts with initializing the PMC card, reading the data log file which contains the information of the last setting device configurations and resetting the controller like the new modified control system software. After writing the position of collimators, pinholes, detector, beam stopper, and sample changer to the controller, the software acquires the neutron counts for 10 seconds to calculate the incoming neutron rates, in order to approximate the consuming time of the experiment.

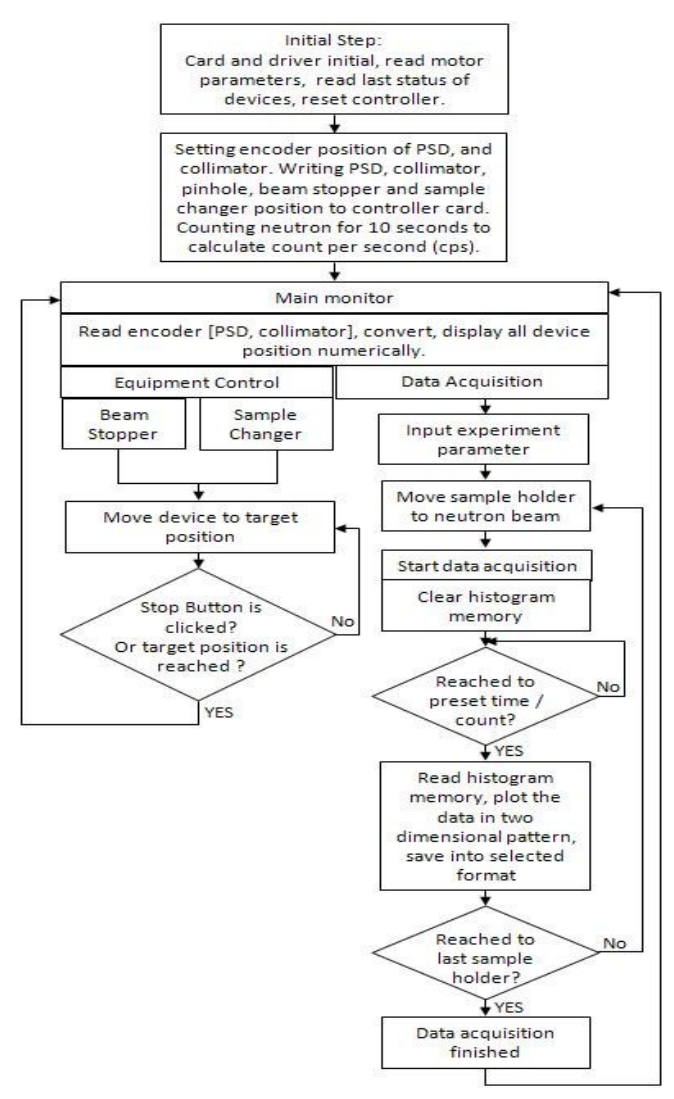

Fig. 5. Flowchart of the SMARTerdata acquisition system software implemented at the computer data acquisition system.

The next step is displaying a main monitor window, measurement and control window of the data acquisition software, Fig. 6(a). All status of the devices are displayed in the main window where several control buttons to handle the beam stopper position, to select the data acquisition mode, and to input the experiment parameters are provided. The input experiment parameters are listed in the menu of experiment parameter windows and very important for the data acquisition before acquiring the neutrons, Fig. 6(b). The modified input parameters include the sample changer parameters for three different types that can be selected for experiment. Since the sample changer accommodates up to 12 sample holders at different positions, the new modified data acquisition software conducts the experiment running automatically and continuously for many samples in sequence without any operator involvement. Therefore the experiment can be executed more effectively and efficiently compared to the one with the previous control and data acquisition systems. The experimental data is periodically stored in two banks of histogram memory alternately in the histogram memory modules. The software takes that stored data every 10 seconds, saves to the selected data format for further analysis, and then displays to the main monitor window in two dimensional patterns.

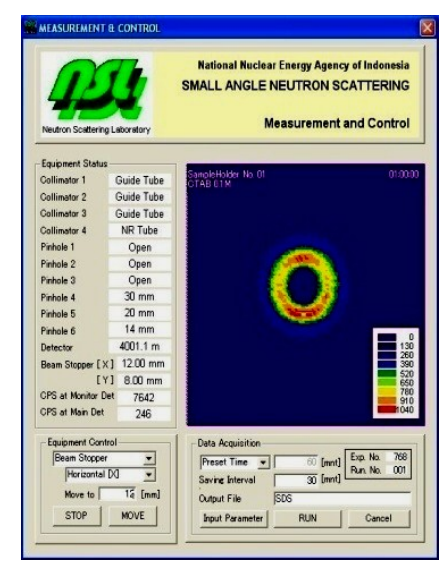

(a)

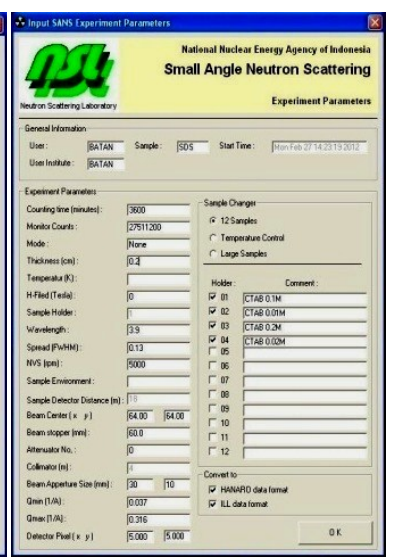

(b)
Fig. 6. The Graphical User Interface (GUI) in (a) main monitor window and (b) input parameters window of the SMARTer data acquisition control system.

\section{RESULTS AND DISCUSSION}

The advantage of the implementation of this new main control and data acquisition systems has been demonstrated on many SANS experiments on various samples. For instance, setting up configuration of the collimation length from $4 \mathrm{~m}$ to $18 \mathrm{~m}$ where it should change 3 collimator tubes, 6 pinhole discs and main detector position can be completed only in 30 minutes as all motor controllers run simultaneously. This new system reduces the consuming time for instrument configuration before performing the experiment by 6 times faster than the previous system. 


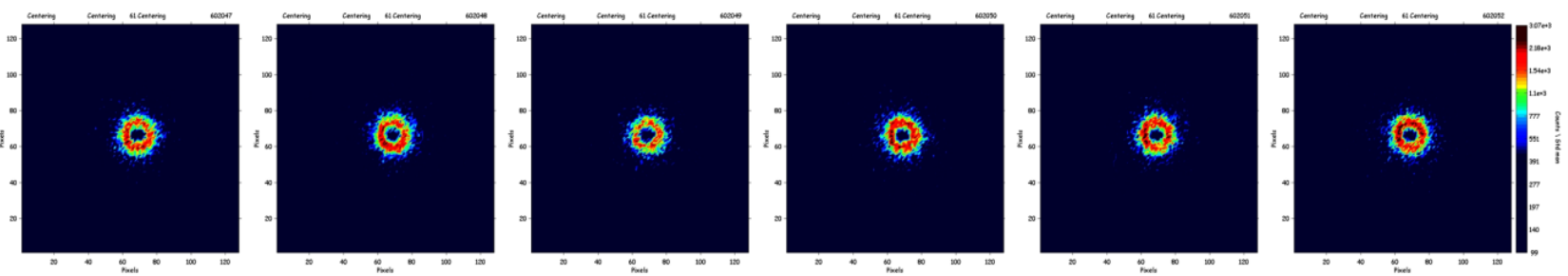

Fig. 7. The sequences of measurement on magnetic powder sample using an automatic temperature-dependent sample changer (6 positions) displayed using the data reduction software analysis. The results show the sample position aligned with the incident beam.

Measurement of magnetic powder $\mathrm{Fe}_{3} \mathrm{O}_{4}$ samples with $1 \mathrm{~mm}$ thick has been conducted automatically in sequence at 6 positions of the automatic temperature-dependent sample changer. This experiment is also confirming the centering position of each sample positions. The result is given in Fig. 7 showing that the incident beam aligned with each sample positions as isotropic scattering from the sample was clearly obtained. Meanwhile, an in-situ experiment on micellar solutions of $0.35 \mathrm{M}$ cetyltrimethylammonium bromide (CTAB) mixed with $0.03 \mathrm{M}$ non-ionic surfactant Triton $\mathrm{X}-100$ solution had also been performed. The experiments were conducted at $15,25,40$ and $60^{\circ} \mathrm{C}$ by regulating the temperature control of the closed-cycle circulator oil bath. The temperature control is now being developed to be regulated by the data acquisition computer as there are still 3 axes available for additional control devices. The 1-dimensional SANS scattering profile of this experiment is given in Fig. 8 and it shows the micellar structural change as a function of temperature.

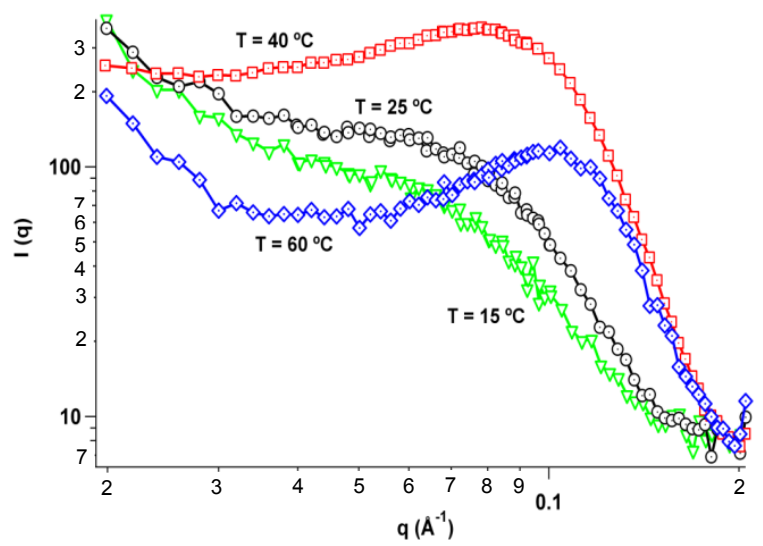

Fig. 8. The 1-dimensional SANS profiles of micellar solution of $0.35 \mathrm{M}$ cetyltrimethylammonium bromide (CTAB) mixed with $0.03 \mathrm{M}$ non-ionic surfactant Triton $\mathrm{X}-100$ as a function of temperature. The data was taken by in-situ experiment.

\section{CONCLUSION}

The SANS BATAN spectrometer, SMARTer has been developed for improving its performance. The recent major developments are as follow (1)
Upgrading the control system for handling all the motor control devices using programmable motor controller for simultaneous movements (2) Implementing up to 12 sample positions of the sample changer for automatic sequential measurement (3) Improving the new user-friendly data acquisition system with some application modes (4) Initiating a stopped-flow system for kinetics study. As described above, the spectrometer is now remarkably more powerful, useful and effective for accommodating more users running the experiment.

\section{ACKNOWLEDGMENT}

This work was supported in part by BATAN under the RSG-GAS Neutron Scattering Facility Development for Materials Characterization (in-house) Project 2010 - 2011 and the Ministry of Research and Technology of Republic of Indonesia, through an Incentive Research Program, Research Grant No. RT2011-2746.

\section{REFERENCES}

1. T. Shinohara, S. Takata, J. Suzuki, et al., Nucl. Instrum. Methods Phys. Res. A 600 (2009) 111.

2. H. Iwase, H. Endo, M. Katagiri, et al., J. Appl. Crystallogr. 44 (2011) 558.

3. G.D. Wignall, K.C. Littrell, W.T. Heller, et al., J. Appl. Crystallogr. 45 (2012) 990.

4. Marsongkohadi and Ridwan, Neutron News 7 (1996) 12.

5. E.G.R. Putra, A. Ikram, E. Santoso, et al., J. Appl. Crystallogr. 40 (2007) s447.

6. E.G.R. Putra, Bharoto, E. Santoso, et al., Nucl. Instrum. Methods Phys. Res. A 600 (2009) 198.

7. E.G.R. Putra, Bharoto and B.S. Seong, J. Phys.: Conf. Ser. 247 (2010) 012010. 
8. E.G.R. Putra and A. Ikram, Nucl. Instrum. Methods Phys. Res. A 600 (2009) 288.

9. E.G.R. Putra, B.S. Seong, E. Shin, et al., J. Phys.: Conf. Ser. 247 (2010) 012028.
10. T. Puspitasari, K.M.L. Raja, D.S. Pangerteni, et al., Procedia Chem. 4 (2012) 186.

11. Bharoto and E.G.R. Putra, AIP Conf. Proc. 1244 (2010) 204. 